symptoms were high temperature in $66.6 \%$, abdominal pain in $29.6 \%$, nausea $14.8 \%$, burn during urination in $18.5 \%$, frequent urination $11.1 \%$, swelling $7.4 \%$, back pain in the $7.4 \%$ of cases. Dominated urine casts were leukocytes, proteins were positive, while bacteria and erythrocytes were positive only to preschool age. Kidney ultrasound resulted normal in $18.5 \%$ of cases, $37 \%$ had pyelonephritic changes. While the sign of urinary stasis $40.7 \%$ of cases (up to the school age) and $11.1 \%$ (school age).

Conclusions In cases with high temperature should be planned examination of urine sediment and an ultrasound examination of abdominal organs, before we plan any other examination.

\section{PEDIATRICIANS RESPONSIBLE FOR EARLY DETETCTION AND SURVELLIANCE OF URINARY TRACT INFECTIONS FROM INFANTS TO PRESCHOOL CHILDREN}

doi:10.1136/archdischild-2012-302724.0892

'S Smugreska, ${ }^{2} \mathrm{~V}$ Tasic, ${ }^{3} \mathrm{D}$ Georgievska, ${ }^{3} \mathrm{~S}$ Spirovska. ${ }^{1}$ Paediatrics, PZU Dr. Svetlana Smugreska; ${ }^{2 N e p h r o l o g y, ~ C l i n i c ~ f o r ~ C h i l d r e n ' s ~ D e s e a s e ; ~}{ }^{3 P e d i a t r i c ~ O f f i c e ~ D r a c e v o, ~}$ Skopje, FYR Macedonia

Introduction Urinary tract infections (UTI) represent substantial pathology of children's morbidity. The frequency is just behind respiratory tract infections. The symptoms may be very diverse and non-specific. Early diagnosis very important for preventing complications (especially renal scarring).

Objectives Pediatrician is the first one that has contact with child having UTI. According to the age, symptomatology is diverse and detection has to be well-timed in order to assure proper treatment. Materials and examinationsIn the study we evaluated 35 children aged 6 months to 6 years (from January 2009 till January 2012). There were 28 female and 7 male children divided in two groups: A) from 6 months till 3 years (20 children)with following symptoms: high temperature, diarrhea, vomiting and lack of appetite. B) from 3-6 years old (15 children) with following symptoms: dysuria, frequency, lumbar/abdominal pain and temperature. Basic laboratory tests and imaging studies were performed: complete blood count, urinalysis, CRP, urineculture, kidney and bladder ultrasound, Tc99mDMSA scan and cystography. According to the results of these studies the children were given appropriate management particularly those with risk for renal scarring.

Conclusion In $86 \%$ of the children with UTI Esherichia Coli (flagelarris) was found, Proteus mirabilis in 6\%, Enteroccocus in 5\% and Staphilococcus 3\%. If the first UTI episode is appropriately managed, children at risk may be selected (high grade VUR) and long term treatment strategy created in order to prevent permanent kidney damage.

\section{BACTERIAL MENINGITIS IN CHILDREN}

doi:10.1136/archdischild-2012-302724.0893

'M Rahimkhani, ${ }^{2 H}$ Khavari Daneshvar. ' ${ }^{1}$ Faculty of Allied Medical Sciences, Tehran University of Medical Science; ${ }^{2}$ Cancer Research Centre, Tehran University of Medical Sciences, Tehran, Iran

Background Meningitis is the most dangerous disease in children and remained irreversible mental disorders. $\mathrm{H}$. influenzae is a fastidious bacteria and may be under detected because of inadequate techniques for isolation or overuse of antibiotics before with recovery of causative agents in bacterial meningitis.in present study two methods, culture and molecular diagnosis (PCR) apply for isolating H.I from CSF.

Methods DNA was extracted from CSF and probed for the presence of Hib DNA with PCR assay with primer derived from the sequences encoding a capsulation-associated protein; a protein most probably involved in the intracellular transportation of the capsular polysaccharide, and would be expected to react only with capsulate $H$. influenzae strains. Primers sequencing were:

Primer 1: 5'- CGT TTG TAT GAT GTT GAT CCA GAC T Primer 2: 5'- TGT CCA TGT CTT CAA AAT GAT G

Results Two hundred three cerebrospinal fluid (CSF) samples collected consecutively from children(less than 5 years) suffering from meningitis were investigated by PCR. There were all the cases of clinical meningitis admitted to three children hospitals in 18 months duration period.

Discussion Two hundred CSF samples were investigated by PCR. Seven samples were positive by PCR method (5 samples were culture positive and 2 samples were culture negative for Haemophilus influenza). Haemopjilus influenza type $b$ is a agent $17.1 \%$ of bacterial meningitis in children surveyed.

\section{REDUCING THE DURATION OF ANTIBIOTIC COURSE IN A NEONATAL UNIT: RESULTS OF A TWO YEAR AUDIT}

doi:10.1136/archdischild-2012-302724.0894

${ }^{1} \mathrm{~A}$ Pantazidou, ${ }^{2} \mathrm{~K}$ Saeed, 'S Struthers. 'Paediatrics; ${ }^{2}$ Microbiology, Hampshire Hospitals NHS Foundation Trust, Winchester, UK

Background and Aims Neonatal intensive care units (NICU) across the UK use different guidelines for the treatment of neonates at risk of sepsis. However, unless specific symptoms/risk factors for sepsis are present, antibiotics are usually stopped at 48 hours if blood culture $(\mathrm{BC})$ results are negative. We aimed to determine whether it would be safe to stop antibiotics at 36 hours.

Methods We conducted a retrospective audit of all blood cultures over a two-year-period (2009-2011) from neonates at risk of or with suspected sepsis admitted to Winchester NICU - a medium-sized level 2 neonatal unit (3000 deliveries/year).

BC were analysed with the automated BacT ALERT ${ }^{\circledR}$ 3D Signature system, (Biomerieux, Durham, UK), using paediatric blood culture bottles (BacT/ALERT ${ }^{\circledR}$ PF; incubated for a total of 5 days.

Results A total of 402 BC were identified and included in the analysis. Eighteen were positive (4.4\%). The median time to BC positivity was 14.5 hours $\left(25^{\text {th }}-75^{\text {th }}\right.$ percentile: $11.5-21.5$ hours). There was no significant difference between the proportion of positive $\mathrm{BC}$ results at 36 and 48 hours ( $p=0.4857$; odds ratio 0.178 (95\% CI: 0.008-3.995)). Only two BC were positive after 36 hours; both were considered to be contaminants and did not changed management.

Conclusions Our data suggest that it is safe, in similar units using similar methods, to stop antibiotics after 36 hours if BC are negative. This would result in a substantial reduction in antibiotic use, invasive procedures and admission time, and thereby has significant implications for neonatal care.

\section{EFFECT OF 4\% CHLORHEXIDINE CORD CLEANSING ON COLONISATION AND BACTERIAL COUNT IN HOSPITAL BORN NEONATES}

doi:10.1136/archdischild-2012-302724.0895

${ }^{1} \mathrm{~S}$ Nangia, ${ }^{1} \mathrm{~A}$ Saili, ${ }^{2} \mathrm{~S}$ Madhesiya, ${ }^{2} \mathrm{P}$ Dhingra, ${ }^{3} \mathrm{U}$ Dhingra, ${ }^{2} \mathrm{~A}$ Dutta, ${ }^{4} \mathrm{VP}$ Menon, ${ }^{3}$ RE Black, ${ }^{2} \mathrm{~S}$ Gupta, ${ }^{2} \mathrm{~A}$ Ahmed, ${ }^{2,3} \mathrm{~S}$ Sazawal. ${ }^{1}$ Department of Pediatrics, Lady Hardinge Medical College \& Kalawati Saran Children's Hospital; ${ }^{2}$ Center for Public Health Kinetics, New Delhi, India; IInternational Health, Johns Hopkins Bloomberg School of Public Health, Baltimore, Virgin Islands (U.S.); ${ }^{4}$ Center for Micronutrient Research, Clinical Trials and Operational Research in Maternal and Child Health (CMR-CTORMCH), Annamalai, India

Background Infections in new-borns are the single most important cause for neonatal mortality in developing countries. Of topical antiseptics chlorhexidine has shown potential as an effective cord care agent. Results from randomized double-blind trials examining the effect of chlorhexidine in Asia have been encouraging 
A $4 \%$ chlorhexidine solution is not comercially available we contracted Galantic Pharma to prepare supplies for two large clinical trials in Africa. This study aimed to evaluate the effect this $4 \%$ chlorhexidine solution on the colonization and colony counts in hospital born infants.

Methods Newborns ( $n=247$ ) from normal deliveries at a hospital in New Delhi were randomly assigned to one of three groups chlorhexidine, placebo or dry cord care. Swab samples were used to collect smear samples before, after $2 \mathrm{hrs}$ and $48 \mathrm{hrs}$ of application of chlorhexidine and at same times from the dry cord group. All swabs were analysed for growth and colony counts.

Results The overall baseline positivity was 20\% (50 of 247 swabs). Chlorhexidine reduced colonisation and bacterial counts in both ( $2 \mathrm{hr}$ and $48 \mathrm{hr}$ ) samples. As compared to placebo and dry cord, the reduction in positivity in chlorhexidine group in 2-hour samples was $80 \%$ [odds ratio of $0.20, p=0.001$ and odds of $0.19, p=0.00$ respectively]. In 48-hour post intervention, chlorhexidine significantly reduced colonisation in comparison to placebo [difference in mean of $-1.01, p=0.006$ ] and dry cord [difference in mean -1.16 , $\mathrm{p}=0.004]$.

Conclusion Cord cleaning with 4\% Chlorhexidine soon after birth reduces colonisation as well as density of pathogens significantly.

\section{VITAMIN D SUPPLEMENTATION AND THE RISK OF INFECTIONS IN FULLTERM INFANTS. CORRELATIONS WITH THE MATERNAL SERUM VITAMIN D}

doi:10.1136/archdischild-2012-302724.0896

AAM El-Mazary, M Abdel-Maaboud, M Mohamed, K Nasef. Minia University Hospital, Minia City, Egypt

Vitamin D insufficiency has been linked with susceptibility to infection, autoimmune diseases and cancers.

Objective To study the effect of vitamin D supplementation on the immunity and the risk of infections during the first year of life in full term infants.

Methods This is a prospective case control study included 99 full term infants attending Minia university hospital during the period from January 2010 to February 2012. They were divided into two groups: group I included forty-eight full term infants supplemented with daily 400 I.U vitamin D for 6-months after birth and group II included fifty-one full term infants not supplemented with vitamin D. History taking and clinical examination and other investigations needed for diagnosis of respiratory, GIT and urinary tract infections were addressed every visit for one year.

Results The incidences of infections totally were less common in infants supplemented with vitamin D than those not supplemented ( $p$-value $=0.01)$. Otitis media, bronchiolitis, pneumonia and gastroenteritis ( $\mathrm{p}$-value $=0.003,0.001,0.001$ and 0.01 respectively) were less common in them. There were no significant differences as regards urinary tract infections between the tow groups ( $p=0.3$ ). significant negative correlations between respiratory and GIT infections and the maternal vitamin $\mathrm{D}$ levels were present.

Conclusions Vitamin D supplementation decreased the incidence of infections especially respiratory and GIT infections. Maternal vitamin $\mathrm{D}$ levels correlated negatively with the incidence of infections.

\section{ROLE OF URINARY TRACT INFECTIONS IN NEONATAL INDIRECT HYPERBILIRUBINEMIA}

doi:10.1136/archdischild-2012-302724.0897

${ }^{1} \mathrm{E}$ Bahat Özdoğan, ${ }^{2} \mathrm{M}$ Mutlu, ${ }^{1} \mathrm{M}$ Kalyoncu, ${ }^{3} \mathrm{G}$ Karagüzel, ${ }^{2} \mathrm{Y}$ Aslan. ${ }^{1}$ Pediatric Nephrology; ${ }^{2}$ Neonatology; ${ }^{3}$ Pediatric Endocrinology, Karadeniz Technical University, Faculty of Medicine, Trabzon, Turkey
Urinary infections are an important cause of prolonged jaundice. But there is conflict about the role of the urinary infections on the pathological jaundice in the first 14 days of the life. This study aims to determine the frequency of urinary tract infections in neonates presenting with jaundice in the first 2 weeks of life with bilirubin levels that require phototherapy.

This study was done with neonates $2-14$ days old they have indirect bilirubin levels above the phototherapy limit but were not found to have any condition that would lead to elevated bilirubin levels, e.g. systemic infection, isoimmunization, erythrocyte enzyme defect, erythrocyte structural defect, hypothyroidism, sequestrated blood, polycythemia, or metabolic disease. Urine samples for urinalysis and culture were obtained using catheterization.

During the study, 482 neonates presented with jaundice and 262 of these fulfilled our criteria. UTI rate was $12 \%$. Mean bilirubin level was $20.9+6.1 \mathrm{mg} / \mathrm{dl}$. Thirtyfive $(13 \%)$ of these patients underwent to blood exchange, the rest were treated with phototherapy only. Weight loss in terms of percentage of birth weight was higher on uninfected patients and rebound bilirubin levels was higher on UTI group.

UTIs may present with isolated jaundice and may cause urosepsis, renal scarring, hypertension and chronic renal failure if they are not treated. In the neonatal period, infections lead to hyperbilirubinemia via hemolysis, inadequate conjugation, decreased excretion and oxidant stres. The findings of this study show the benefits of obtaining urine cultures for the diagnosis of UTI in neonatal patients with hyperibilirubinemia requiring phototherapy who have unexplained hyperbilirubinemia.

\section{REDUCING NEONATAL INFECTIONS IN SOUTH AND SOUTHERN CENTRAL VIETNAM: THE OPINION OF HEALTH CAREGIVERS}

doi:10.1136/archdischild-2012-302724.0898

'D Trevisanuto, 'V Dal Cengio, ' 2 Thi Ahn Thu, ${ }^{3} \mathrm{G}$ Arnolda, ${ }^{3} \mathrm{~T}$ Dihn Chien, ${ }^{3} \mathrm{D}$ Kumara, ${ }^{3} \mathrm{~L}$ Moccia, ${ }^{4} \mathrm{~N}$ Mihn Xuan. ${ }^{1}$ Children and Women's Health Department, Padua University School of Medicine, Padova, Italy; ${ }^{2}$ Cho Ray Hospital, Hochiminh City, Vietnam; ${ }^{3}$ East Meets West Foundation, Oakland, CA, USA; ${ }^{4}$ Tu Du Hospital, Hochiminh City, Vietnam

Background Infection represents a consistent cause of neonatal mortality in high as well as low income countries. In this study, we assessed the opinion of healthcare providers on how to improve neonatal infection control and prevention in South and Southern Central Vietnam hospitals.

Methods Fifty-four participants to a workshop on infection control and prevention were asked to fill out an anonymous, written questionnaire regarding the priorities that could improve neonatal infection control and prevention in provincial hospitals in South and Southern Central Vietnam.

Results Hand washing, exclusive breastfeeding and safe disposal of medical waste were scored by participants as the highest priorities for preventing neonatal infections. Education through instructional posters and written guidelines, family contact, kangaroo-mothercare, limitation of invasive procedures and screening for maternal GBS infection received relatively low scores.

Conclusions The opinions of operators involved in neonatal health match international recommendations for infection prevention with regards to some, but not all, issues, suggesting the need for a better understanding of this phenomenon. Our results may contribute to better design interventions for infection prevention in settings with limited resources.

\section{NEWBORNS AND INFECTIOUS RISKS:EXPERIENCE OF MATERNITY HOSPITAL SOUISSI-RABAT}

doi:10.1136/archdischild-2012-302724.0899 